\title{
Octonion Analysis of Several Variables
}

\author{
Haiyan Wang • Guangbin Ren
}

Received: 22 May 2014 / Accepted: 10 September 2014 / Published online: 11 October 2014

(C) School of Mathematical Sciences, University of Science and Technology of China and Springer-Verlag Berlin Heidelberg 2014

\begin{abstract}
The octonions are distinguished in the $M$-theory in which Universe is the usual Minkowski space $\mathbb{R}^{4}$ times a $G_{2}$ manifold of very small diameter with $G_{2}$ being the automorphism group of the octonions. The multidimensional octonion analysis is initiated in this article, which extends the theory of several complex variables, such as the Bochner-Martinelli formula, the theory of non-homogeneous Cauchy-Riemann equations, and the Hartogs principle, to the non-commutative and non-associative realm.
\end{abstract}

Keywords Several octonionic variables · Bochner-Martinelli formula . Hartogs theorem · Non-homogenous Cauchy-Riemann equations

Mathematics Subject Classification $35 \mathrm{G} 20 \cdot 30 \mathrm{G} 35 \cdot 32 \mathrm{G} 05$

\section{Introduction}

The importance of the octonions has been found in string theory, special theory of relativity, and quantum theory $[3,4]$ since the birth of the octonions discovered in 1843 by Graves and constructed in 1845 by Cayley. It is known that the automorphism group of the octonion algebra is the exceptional simple Lie group $G_{2}$, while the $M$ theory claims that the model of Universe is the usual Minkowski space $\mathbb{R}^{4}$ times a $G_{2}$ manifold of very small diameter.

\section{H. Wang}

School of Science, Tianjin University of Technology and Education, Tianjin 300222, China e-mail: whaiyan@mail.ustc.edu.cn

G. Ren $(\bowtie)$

Department of Mathematics, University of Science and Technology of China, Hefei 230026, China e-mail: rengb@ustc.edu.cn 
The octonion analysis is quite subtle due to non-commutative and non-associative feature. It is only restricted to the setting of one octonionic variable and is in its primary stage [13].

Comparing to the recently well-developed theory of several quaternionic theory for Cauchy-Fueter operators $[5,7,16]$, nearly nothing have been done about multidimensional octonion analysis.

In this paper, we study the octonion analysis of several variables and extend the theory of several complex variables to the octonions. In particular, we shall construct the explicit form of the Bochner-Martinelli integral representation formula related to several Dirac operators, solve the system of non-homogeneous Cauchy-Riemann equations of the octonionic version, and establish the Hartogs theorem on removability of compact singularity for regular functions in the octonion analysis of several variables.

The Bochner-Martinelli integral representation formula is the key in the integral theory of the octonionic analysis of several variables. We refer to $[9,11,12]$ for the version of several variables and its applications.

The solvability of the non-homogeneous Cauchy-Riemann equations will lay the foundation for constructing functions in the octonionic analysis of several variables. As it is well known that many of the differences between the one and several complex variables can be accounted for by the support behavior of solutions of the inhomogeneous Cauchy-Riemann equations [11]. The inhomogeneous Cauchy-Riemann equations are also studied in the setting of quaternions [6,14] and Clifford algebras [15].

The Hartogs phenomena distinguish several octonionic variables from one octonionic variable.

In the study of analysis in the non-commutative realm, there are two approaches. The analytic approach $[14,15]$ has the advantage over the standard one from algebraic geometry $[1,2,5,7,16]$, because it can provide all required functions explicitly. In this article, we shall adopt the latter approach.

\section{Octonion Analysis of One Variable}

In this section, we recall some known classical results in the octonion analysis of one variable and provide a new version of the Cauchy-Pompeiu integral formula for later use.

The octonions $\mathbb{O}$ are the non-associative, non-commutative, normed division algebra over the real generated by $e_{1}, \ldots, e_{7}$. For convenience, we also denote $e_{0}=1$.

In terms of a natural basis, an octonion can be written as

$$
x=x_{0}+\sum_{i=1}^{7} x_{i} e_{i}, \quad x_{i} \in \mathbb{R}
$$

where the basis satisfies the rule

$$
e_{i} e_{j}+e_{j} e_{i}=-2 \delta_{i j}, \quad i, j=1, \ldots, 7 \text {. }
$$


Fig. 1 Fano Mnemonic

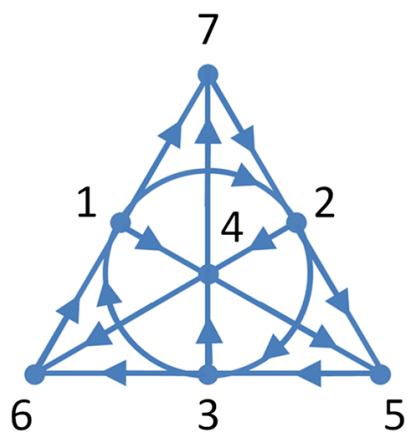

The full multiplication table is conveniently encoded in the 7-point projective plane, shown in Fig. 1. In the Fano mnemonic graph, the vertices are labeled by $1, \ldots, 7$ instead of $e_{1}, \ldots, e_{7}$. Each of the 7 oriented lines gives a quaternionic triple. The product of any two imaginary units is given by the third unit on the unique line connecting them, with the sign determined by the relative orientation.

The associator of three octonions is defined as

$$
[x, y, z]=(x y) z-x(y z)
$$

for any $x, y, z \in \mathbb{O}$, which is totally antisymmetric such as

$$
[x, y, z]=-[y, x, z]=[y, z, x]
$$

in its arguments and has no real part. Although the associator does not vanish in general, the octonions do satisfy a weak form of associativity known as alternativity, namely

$$
[x, x, y]=0
$$

and the so-called R. Monfang identities:

$$
(x y x) z=x(y(x z)), \quad z(x y x)=((z x) y) x, \quad x(y z) x=(x y)(z x) .
$$

The underlying reason for this is that two octonions determine a quaternionic subalgebra of $(\mathbb{O}$, so that any product containing only two octonionic directions is associative.

Any octonion is expressed as the sum of a scalar part $x_{0}$ and a vector part $\underline{x}$, i.e.,

$$
x=x_{0}+\underline{x} .
$$

Octonionic conjugation is given by reversing the sign of the imaginary basis units, i.e.,

$$
\bar{x}=x_{0}-\sum_{i=1}^{7} x_{i} e_{i} .
$$

Conjugation is an antiautomorphism, since it satisfies $\overline{x y}=\bar{y} \bar{x}$. 
For a single octonionic variable, the octonionic Dirac operator is defined as (see [13])

$$
D=\sum_{i=0}^{7} e_{i} \partial_{x_{i}}
$$

More precisely,

$$
\begin{aligned}
& D f(x)=\sum_{i, j=0}^{7} \partial_{x_{i}} f_{j}(x) e_{i} e_{j}, \\
& f(x) D=\sum_{i, j=0}^{7} \partial_{x_{i}} f_{j}(x) e_{j} e_{i}
\end{aligned}
$$

for any $f(x)=\sum_{j=0}^{7} f_{j}(x) e_{j}$ with $f_{j}(x)$ being real valued.

The Stokes formula in octonionic analysis of one variable states that [13]

$$
\int_{\partial \Omega} f(\mathrm{~d} \sigma g)=\int_{\Omega}(f D) g+f(D g)-\sum_{j=0}^{7}\left[e_{j}, D f_{j}, g\right] \mathrm{d} v .
$$

Here, $d v$ is the volume measure and $d \sigma$ the octonion-valued surface measure, given by

$$
\mathrm{d} \sigma_{y}=\sum_{i=0}^{7}(-1)^{i} e_{i} \mathrm{~d} \hat{y}_{i}
$$

where $d \hat{y}_{i}=\mathrm{d} y_{0} \wedge \cdots \wedge \mathrm{d} y_{i-1} \wedge \mathrm{d} y_{i+1} \wedge \cdots \wedge d y_{7}$.

The Cauchy-Pompeiu integral formula in octonionic analysis of one variable takes the form

$$
\chi_{\Omega}(x) f(x)=\int_{\partial \Omega} E(y-x)\left(\mathrm{d} \sigma_{y} f(y)\right)-\int_{\Omega} E(y-x)\left(D_{y} f(y)\right) \mathrm{d} v_{y} .
$$

Here, $\chi_{\Omega}$ is defined as

$$
\chi_{\Omega}(x)= \begin{cases}1, & x \in \Omega, \\ 1 / 2, & x \in \partial \Omega, \\ 0, & x \neq \bar{\Omega},\end{cases}
$$

and $E$ is the Cauchy kernel of the octonionic Dirac operator, i.e.,

$$
E(y-x)=\frac{1}{\omega_{8}} \frac{\overline{y-x}}{|y-x|^{8}}
$$

where $\omega_{8}=\pi^{4} / 3$ is the area of the unit sphere in $\mathbb{R}^{8}$. When $x \in \partial \Omega$, we always interpret the singular integral in (2.3) as its Cauchy principal value, i.e., 


$$
\int_{\partial \Omega} E(y-x)\left(\mathrm{d} \sigma_{y} f(y)\right)=\lim _{\epsilon \rightarrow 0+} \int_{\partial \Omega \backslash B(x, \epsilon)} E(y-x)\left(\mathrm{d} \sigma_{y} f(y)\right) .
$$

We can now provide a new version of the Cauchy-Pompeiu integral formula.

Lemma 2.1 Let $U$ be an open set in $\mathbb{R}^{8}$, and let $\Omega$ be a domain with differentiable boundary such that $\Omega$ is relatively compact in $U$. If $g \in C^{1}(U, \mathbb{O})$, then

$$
\chi_{\Omega}(x) g(x)=\int_{\partial \Omega} d \sigma_{y}(E(y-x) g(y))-\int_{\Omega} \sum_{i=0}^{7} e_{i}\left(E(y-x) \partial_{y_{i}} g(y)\right) \mathrm{d} v_{y} .
$$

Proof We first claim that

$$
D_{y}(E(y-x) g(y))=\left(D_{y} E(y-x)\right) g(y)+\sum_{i=0}^{7} e_{i}\left(E(y-x)\left(\partial_{y_{i}} g(y)\right)\right) \text {. }
$$

Indeed, by definition we have

$$
D_{y}(E(y-x) g(y))=\sum_{i=0}^{7} e_{i}\left(\partial_{y_{i}} E(y-x) g(y)\right)+\sum_{i=0}^{7} e_{i}\left(E(y-x)\left(\partial_{y_{i}} g(y)\right)\right)
$$

By the definitions of the associator and the Dirac operator, the first term on the right side above equals

$$
\sum_{i=0}^{7}\left(e_{i} \partial_{y_{i}} E(y-x)\right) g(y)-\sum_{i=0}^{7}\left[e_{i}, \partial_{y_{i}} E(y-x), g(y)\right]
$$

To prove the claim, we need only to show

$$
A:=\sum_{i=0}^{7}\left[e_{i}, \partial_{y_{i}} E(y-x), g(y)\right]=0 .
$$

We now rewrite $E(y-x)$ as

$$
E(y-x)=\sum_{j=0}^{7} E^{j}(y-x) e_{j}
$$

where

$$
E^{0}(y-x)=\frac{y_{0}-x_{0}}{\omega_{8}|y-x|^{8}}, \quad E^{j}(y-x)=-\frac{y_{j}-x_{j}}{\omega_{8}|y-x|^{8}} .
$$


By calculation,

$$
\partial_{y_{i}} E^{j}(y-x)=-\frac{\delta_{i j}}{\omega_{8}|y-x|^{8}}+\frac{8\left(y_{i}-x_{i}\right)\left(y_{j}-x_{j}\right)}{\omega_{8}|y-x|^{10}}
$$

for any $i, j=1, \ldots, 7$.

Since the associator $[x, y, z]$ vanishes provided one of $x, y, z$ being $e_{0}$, it follows from (2.7) that

$$
\begin{aligned}
A & =\sum_{i=0}^{7}\left[e_{i}, \sum_{j=0}^{7} \partial_{y_{i}} E^{j}(y-x) e_{j}, g(y)\right] \\
& =\sum_{i=1}^{7}\left[e_{i}, \sum_{j=1}^{7} \partial_{y_{i}} E^{j}(y-x) e_{j}, g(y)\right] \\
& =\frac{1}{\omega_{8}} \sum_{i=1}^{7}\left[e_{i}, \sum_{j=1}^{7}\left(-\frac{\delta_{i j}}{|y-x|^{8}}+\frac{8\left(y_{i}-x_{i}\right)\left(y_{j}-x_{j}\right)}{|y-x|^{10}}\right) e_{j}, g(y)\right]
\end{aligned}
$$

and thus by the linearity of associator, we obtain

$$
A=\frac{1}{\omega_{8}} \sum_{i=1}^{7} \sum_{j=1}^{7}\left\{\left[e_{i},-\frac{\delta_{i j}}{|y-x|^{8}} e_{j}, g(y)\right]+\left[e_{i}, \frac{8\left(y_{i}-x_{i}\right)\left(y_{j}-x_{j}\right)}{|y-x|^{10}} e_{j}, g(y)\right]\right\}
$$

The first term in the brackets above vanishes due to the property of associator $[x, x, y]=0$. This means

$$
\begin{aligned}
A & =\frac{1}{\omega_{8}} \sum_{i=1}^{7} \sum_{j=1}^{7}\left[e_{i}, \frac{8\left(y_{i}-x_{i}\right)\left(y_{j}-x_{j}\right)}{|y-x|^{10}} e_{j}, g(y)\right] \\
& =\frac{1}{\omega_{8}} \frac{8}{|y-x|^{10}}[\underline{y-x}, \underline{y-x}, g(y)]=0
\end{aligned}
$$

and finishes the proof of the claim.

From the claim,

$$
D_{y}(E(y-x) g(y))=\sum_{i=0}^{7} e_{i}\left(E(y-x)\left(\partial_{y_{i}} g(y)\right)\right)
$$

for any $x \neq y$. 
If $x \in U \backslash \bar{\Omega}$, then applying the Stokes theorem and formula (2.8), we get

$$
\begin{aligned}
\int_{\Omega} \sum_{i=0}^{7} e_{i}\left(E(y-x)\left(\partial_{y_{i}} g(y)\right)\right) \mathrm{d} v_{y} & =\int_{\Omega} D_{y}(E(y-x) g(y)) \mathrm{d} v_{y} \\
& =\int_{\partial \Omega} \mathrm{d} \sigma_{y}(E(y-x) g(y)) .
\end{aligned}
$$

This proves (2.5) in the case of $x \notin \Omega$.

In order to prove (2.5) in the case of $x \in \Omega$, again we apply the Stokes theorem and formula (2.8) to deduce that

$$
\int_{\Omega \backslash B(x, \epsilon)} \sum_{i=0}^{7} e_{i}\left(E(y-x)\left(\partial_{y_{i}} g(y)\right)\right) \mathrm{d} v_{y}=\left\{\int_{\partial \Omega}-\int_{\partial B(x, \epsilon)}\right\} \mathrm{d} \sigma_{y}(E(y-x) g(y)) .
$$

By taking the limit process $\epsilon \rightarrow 0$ in the above formula, it is sufficient to show

$$
\begin{aligned}
\lim _{\epsilon \rightarrow 0} \int_{B(x, \epsilon)} \sum_{i=0}^{7} e_{i}\left(E(y-x)\left(\partial_{y_{i}} g(y)\right)\right) \mathrm{d} v_{y} & =0 \\
\lim _{\epsilon \rightarrow 0} \int_{\partial B(x, \epsilon)} \mathrm{d} \sigma_{y}(E(y-x) g(y)) & =g(x) .
\end{aligned}
$$

Since $\partial_{y_{i}} g(y)$ is bounded, it is evident that $(2.10)$ holds true. It remains to show (2.11).

For any $y \in \partial B(x, \epsilon)$, we have

$$
|g(y)-g(x)| \leq|x-y| \sup _{\zeta \in \overline{B(x, \epsilon)}}|\nabla g(\zeta)|
$$

so that

$$
\lim _{\epsilon \rightarrow 0} \int_{\partial B(x, \epsilon)} \mathrm{d} \sigma_{y}(E(y-x)(g(y)-g(x)))=0 .
$$

Notice that

$$
\mathrm{d} \sigma_{y}=\frac{y-x}{|y-x|} \mathrm{d} s_{y}
$$

for any $y \in \partial B(x, \epsilon)$, where $d s_{y}$ is the surface element of the sphere $\partial B(x, \epsilon)$. This together with (2.4) implies

$$
\left[\mathrm{d} \sigma_{y}, E(y-x), g(x)\right]=0
$$


so that

$$
\mathrm{d} \sigma_{y}(E(y-x) g(x))=\left(\mathrm{d} \sigma_{y} E(y-x)\right) g(x) .
$$

Therefore,

$$
\begin{aligned}
\int_{\partial B(x, \epsilon)} \mathrm{d} \sigma_{y}(E(y-x) g(x)) & =\int_{\partial B(x, \epsilon)}\left(\mathrm{d} \sigma_{y} E(y-x)\right) g(x) \\
& =\frac{1}{\omega_{8} \epsilon^{7}} \int_{\partial B(x, \epsilon)} \mathrm{d} s_{y} g(x) \\
& =g(x) .
\end{aligned}
$$

This proves (2.11) so that (2.5) holds true in the case of $x \in \Omega$.

The case of $x \in \partial \Omega$ can be proved similarly.

As a consequence of the Cauchy-Pompeiu integral formula above as well as the classical one, we have the following formula useful in the proof of the existence of the solution of Dirac systems with compact support (see Sect. 4).

Lemma 2.2 Let $U$ be an open set in $\mathbb{R}^{8}$, and let $\Omega$ be a domain with differentiable boundary such that $\Omega$ is relatively compact in $U$. If $f \in C^{1}(U, \mathbb{O})$, then for any $j=0,1, \ldots, 7$,

$$
\begin{gathered}
\int_{\Omega} e_{j}\left\{E(y-x)\left(D_{y} f(y)\right)\right\} d v_{y}-\int_{\Omega} \sum_{i=0}^{7} e_{i}\left\{E(y-x)\left(e_{j} \partial_{y_{i}} f(y)\right)\right\} d v_{y} \\
=\int_{\partial \Omega} e_{j}\left\{E(y-x)\left(d \sigma_{y} f(y)\right)\right\}-\int_{\partial \Omega} d \sigma_{y}\left\{E(y-x)\left(e_{j} f(y)\right)\right\} .
\end{gathered}
$$

Proof The result follows when we equal the two formulas, in which one is (2.5) with $g(y)=e_{j} f(y)$ and the other is (2.3) multiplied by $e_{j}$ from left in both sides.

\section{The Bochner-Martinelli Formula}

In this section, we shall establish the Bochner-Martinelli formula in several octonionic variables.

\subsection{The Bochner-Martinelli Kernel}

In $\mathbb{O}^{n} \simeq \mathbb{R}^{8 n}$, we denote

$$
x=\left(x_{1}, x_{2}, \ldots, x_{n}\right) \in \mathbb{O}^{n}
$$

with

$$
x_{l}=\left(x_{l 0}, x_{l 1}, \ldots, x_{l 7}\right) \in \mathbb{R}^{8} \simeq \mathbb{O} \text {. }
$$


We now introduce a system of Dirac operators

$$
D_{l}=\sum_{i=0}^{7} e_{i} \partial_{x_{l i}}, \quad l=1,2, \ldots, n
$$

Definition 3.1 Let $U$ be an open set in $\mathbb{O}^{n}$ and $f \in C^{1}(U, \mathbb{O})$. We say that $f$ is (left) octonionic regular in $U$ if

$$
D_{1} f=\cdots=D_{n} f=0 .
$$

We can now introduce the Bochner-Martinelli kernel in several octonionic variables. With the notation in (3.1) and (3.2), we define

$$
K_{B M}(y-x)=\sum_{l=1}^{n} E_{l}(y-x) d \Sigma_{y_{l}},
$$

where

$$
E_{l}(y-x)=\frac{1}{\omega_{8 n}} \frac{\overline{y_{l}-x_{l}}}{|y-x|^{8 n}},
$$

and $\mathrm{d} \Sigma_{y_{l}}$ is the octonionic-valued surface measure

$$
\mathrm{d} \Sigma_{y_{l}}=\mathrm{d} v_{y_{1}} \wedge \mathrm{d} v_{y_{2}} \wedge \cdots \wedge \mathrm{d} v_{y_{l-1}} \wedge \mathrm{d} \sigma_{y_{l}} \wedge \mathrm{d} v_{y_{l+1}} \wedge \cdots \wedge \mathrm{d} v_{y_{n}}
$$

Here the volume and surface measure in the $l$-th copy of $\mathbb{O}$ in $\mathbb{O}^{n}$ are denoted by $d v_{y_{l}}$ and $d \sigma_{y_{l}}$, respectively. More explicitly,

$$
\begin{aligned}
\mathrm{d} v_{y_{l}} & =\mathrm{d} y_{l 0} \wedge \mathrm{d} y_{l 1} \wedge \cdots \wedge \mathrm{d} y_{l 7}, \\
\mathrm{~d} \sigma_{y_{l}} & =\sum_{j=0}^{7}(-1)^{j} e_{j} \mathrm{~d} \widehat{y_{l j}}, \\
\mathrm{~d} \widehat{y_{l j}} & =\mathrm{d} y_{l 0} \wedge \cdots \wedge \mathrm{d} y_{l(j-1)} \wedge \mathrm{d} y_{l(j+1)} \wedge \cdots \wedge \mathrm{d} y_{l 7} .
\end{aligned}
$$

The constant $\omega_{8 n}$ stands for the area of the unit sphere in $\mathbb{R}^{8 n}$.

We shall know that the kernel $K_{B M}(y-x)$ is exactly the Bochner-Martinelli kernel of octonion analysis of several variables.

We remark that if $n=1$, the kernel $K_{B M}(y-x)$ turns out to be the classical Cauchy kernel in octonion analysis of one variable [13].

\subsection{Bochner-Martinelli Formula}

Some technique lemmas are needed in order to establish the Bochner-Martinelli formula of several octonionic variables. 
We denote the volume element of $\mathbb{O}^{n}$ as

$$
\mathrm{d} v_{y}=\mathrm{d} v_{y_{1}} \wedge \mathrm{d} v_{y_{2}} \wedge \cdots \wedge \mathrm{d} v_{y_{n}}
$$

with $\mathrm{d} v_{y_{l}}$ being defined as in (3.6).

Lemma 3.2 Let $U$ be an open set in $\mathbb{O}^{n}$ and $g, f \in C^{1}(U, \mathbb{O})$. Then

$$
\mathrm{d}\left(g\left(d \Sigma_{y_{l}} f\right)\right)=\left(\left(g D_{l}\right) f+g\left(D_{l} f\right)-\sum_{q=0}^{7}\left[\partial_{l q} g, e_{q}, f\right]\right) \mathrm{d} v_{y}
$$

for any $l=1,2, \ldots, n$.

Proof Let $l \in\{1, \ldots, n\}$ be fixed. By definition,

$$
d\left(g\left(d \Sigma_{y_{l}} f\right)\right)=\sum_{p=1}^{n} \sum_{q=0}^{7}\left(\left(\partial_{y_{p q}} g\right)\left(\mathrm{d} y_{p q} \wedge d \Sigma_{y_{l}} f\right)+g\left(\mathrm{~d} y_{p q} \wedge \mathrm{d} \Sigma_{y_{l}} \partial_{y_{p q}} f\right)\right) .
$$

To evaluate the right side, we claim that

$$
\mathrm{d} y_{p q} \wedge \mathrm{d} \Sigma_{y_{l}}=\delta_{p l} e_{q} \mathrm{~d} v_{y}
$$

From the claim, we get

$$
\begin{aligned}
\mathrm{d}\left(g\left(d \Sigma_{y_{l}} f\right)\right) & =\sum_{q=0}^{7}\left(\left(\partial_{y_{l q}} g\right)\left(e_{q} f\right)+g\left(\partial_{y_{l q}} e_{q} f\right)\right) \mathrm{d} v_{y} \\
& =\sum_{q=0}^{7}\left(\left(\partial_{y_{l q}} g e_{q}\right) f-\left[\partial_{y_{l q}} g, e_{q}, f\right]+g\left(\partial_{y_{l q}} e_{q} f\right)\right) \mathrm{d} v_{y} \\
& =\left(\left(g D_{l}\right) f+g\left(D_{l} f\right)-\sum_{q=0}^{7}\left[\partial_{y_{l q}} g, e_{q}, f\right]\right) \mathrm{d} v_{y} .
\end{aligned}
$$

It remains to prove the claim. If $p \neq l$, then

$$
\mathrm{d} y_{p q} \wedge \mathrm{d} \Sigma_{y_{l}}=\mathrm{d} y_{p q} \wedge \mathrm{d} v_{y_{1}} \wedge \cdots \wedge \mathrm{d} v_{y_{l-1}} \wedge \mathrm{d} \sigma_{y_{l}} \wedge \mathrm{d} v_{y_{l+1}} \wedge \cdots \wedge \mathrm{d} v_{y_{n}}=0 .
$$

When $p=l$, we know

$$
\begin{aligned}
\mathrm{d} y_{p q} \wedge d \Sigma_{y_{l}} & =\mathrm{d} y_{l q} \wedge \mathrm{d} \Sigma_{y_{l}} \\
& =\mathrm{d} y_{l q} \wedge \mathrm{d} v_{y_{1}} \wedge \cdots \wedge \mathrm{d} v_{y_{l-1}} \wedge \mathrm{d} \sigma_{y_{l}} \wedge \mathrm{d} v_{y_{l+1}} \wedge \cdots \wedge \mathrm{d} v_{y_{n}} \\
& =\mathrm{d} v_{y_{1}} \wedge \cdots \wedge \mathrm{d} v_{y_{l-1}} \wedge \mathrm{d} y_{l q} \wedge \mathrm{d} \sigma_{y_{l}} \wedge \mathrm{d} v_{y_{l+1}} \wedge \cdots \wedge \mathrm{d} v_{y_{n}} \\
& =\mathrm{d} v_{y_{1}} \wedge \cdots \wedge \mathrm{d} v_{y_{l-1}} \wedge \sum_{j=0}^{7}(-1)^{j} e_{j} \mathrm{~d} y_{l q} \wedge \mathrm{d} \widehat{y_{l j}} \wedge \mathrm{d} v_{y_{l+1}} \wedge \cdots \wedge \mathrm{d} v_{y_{n}} .
\end{aligned}
$$


Due to

$$
(-1)^{j} e_{j} \mathrm{~d} y_{l q} \wedge \mathrm{d} \widehat{y_{l j}}=\delta_{q j} e_{j} \mathrm{~d} v_{y_{l}}
$$

the claim follows and this finishes the proof.

Lemma 3.3 For any $x \neq y$,

$$
\mathrm{d}_{y}\left(K_{B M}(y-x)\right)=0
$$

Proof By Lemma 3.2 and the definition of $K_{B M}(y-x)$, we know

$$
\mathrm{d}_{y}\left(K_{B M}(y-x)\right)=\sum_{l=1}^{n}\left(E_{l}(y-x) D_{l}\right) \mathrm{d} v_{y} .
$$

From (3.4),

$$
E_{l}(y-x) D_{l}=\frac{1}{\omega_{8 n}} \sum_{j=0}^{7} \partial_{y_{l j}} \frac{\overline{y_{l}-x_{l}}}{|y-x|^{8 n}} e_{j}
$$

By calculation,

$$
\partial_{y_{l j}} \frac{\overline{y_{l}-x_{l}}}{|y-x|^{8 n}}=\left(\frac{\bar{e}_{j}}{|y-x|^{8 n}}-\overline{y_{l}-x_{l}} \frac{8 n\left(y_{l j}-x_{l j}\right)}{|y-x|^{8 n+2}}\right),
$$

we have

$$
\begin{aligned}
E_{l}(y-x) D_{l} & =\frac{1}{\omega_{8 n}}\left(\frac{8}{|y-x|^{8 n}}-\overline{y_{l}-x_{l}} \frac{8 n\left(y_{l}-x_{l}\right)}{|y-x|^{8 n+2}}\right) \\
& =\frac{1}{\omega_{8 n}}\left(\frac{8}{|y-x|^{8 n}}-8 n \frac{\left|y_{l}-x_{l}\right|^{2}}{|y-x|^{8 n+2}}\right) .
\end{aligned}
$$

This means

$$
\sum_{l=1}^{n}\left(E_{l}(y-x) D_{l}\right)=0
$$

So that from (3.9), we have

$$
\mathrm{d}_{y}\left(K_{B M}(y-x)\right)=0
$$

for any $x \neq y$.

Theorem 3.4 Let $U$ be an open bounded set in $\mathbb{R}^{8 n}$ and $\partial U$ be diffeomorphic to the sphere $S^{8 n-1}$ in $\mathbb{R}^{8 n}$ and assume that $\partial U$ is of class $C^{1}$. Then 


$$
\int_{\partial U} K_{B M}(y-x)=\chi_{U}(x)
$$

Proof When $x \in \mathbb{R}^{8 n} \backslash \bar{U}$, the result follows directly from the Stokes Theorem and Lemma 3.3.

$$
\int_{\partial U} K_{B M}(y-x)=0, \quad x \in \mathbb{R}^{8 n} \backslash \bar{U} .
$$

When $x \in U$, the Stokes theorem can also be applied through digging out the singular point. Therefore, for sufficiently small $\epsilon>0$, we have

$$
\int_{\partial U} K_{B M}(y-x)=\int_{|y-x|=\epsilon} K_{B M}(y-x) .
$$

The right side can be easily calculated. Indeed, by definition we get

$$
\int_{|y-x|=\epsilon} K_{B M}(y-x)=\frac{1}{\omega_{8 n} \epsilon^{8 n}} \sum_{l=1}^{n} \int_{|y-x|=\epsilon} \overline{y_{l}-x_{l}} \mathrm{~d} \Sigma_{y_{l}}
$$

so that the Stokes theorem and Lemma 3.2 yield

$$
\begin{aligned}
\int_{|y-x|=\epsilon} K_{B M}(y-x) & =\frac{1}{\omega_{8 n} \epsilon^{8 n}} \sum_{l=1}^{n} \int_{|y-x| \leq \epsilon} d_{y}\left(\overline{y_{l}-x_{l}} \mathrm{~d} \Sigma_{y_{l}}\right) \\
& =\frac{1}{\omega_{8 n} \epsilon^{8 n}} \sum_{l=1}^{n} \int_{|y-x| \leq \epsilon}\left(\overline{y_{l}-x_{l}} D_{l}\right) \mathrm{d} v_{y} \\
& =\frac{8}{\omega_{8 n} \epsilon^{8 n}} \sum_{l=1}^{n} \int_{|y-x| \leq \epsilon} \mathrm{d} v_{y} \\
& =1 .
\end{aligned}
$$

The case of $x \in \partial \Omega$ can be proved similarly. This finishes the proof.

If the density function is replaced by $f$ instead of 1 , for simplicity, we denote

$$
K_{B M}(y-x) f(y):=\sum_{l=1}^{n} E_{l}(y-x)\left(\mathrm{d} \Sigma_{y_{l}} f(y)\right) .
$$

Now we come to the main result of this section.

Theorem 3.5 [Bochner-Martinelli] Let $U$ be an open bounded set in $\mathbb{R}^{8 n}$ with $C^{2}$ boundary $\partial U$. If $f \in C(\bar{U}, \mathbb{O}) \cap C^{1}(U, \mathbb{O})$, then for any $x \in U$, we have

$$
f(x)=\int_{\partial U} K_{B M}(y-x) f(y)-\sum_{l=1}^{n} \int_{U} E_{l}(y-x)\left(D_{l} f(y)\right) \mathrm{d} v_{y} .
$$


Proof By the Stokes theorem, for any fixed $x \in U$, we have

$$
\left\{\int_{\partial U}-\int_{\partial B(x, \epsilon)}\right\} K_{B M}(y-x) f(y)=\int_{U \backslash B(x, \epsilon)} \mathrm{d}_{y}\left(K_{B M}(y-x) f(y)\right) .
$$

We first calculate the differential form on the right hand. In light of (3.12) and Lemma 3.2 ,

$$
\begin{aligned}
\mathrm{d}_{y}\left(K_{B M}(y-x) f(y)\right)= & \sum_{l=1}^{n}\left(\left(E_{l}(y-x) D_{y_{l}}\right) f(y)+E_{l}(y-x)\left(D_{y_{l}} f(y)\right)\right. \\
& \left.-\sum_{j=0}^{7}\left[\partial_{y_{l j}} E_{l}(y-x), e_{j}, f(y)\right]\right) \mathrm{d} v_{y} .
\end{aligned}
$$

The first item vanishes after summation due to (3.11). We claim that the last term also vanishes, i.e.,

$$
\sum_{l=1}^{n} \sum_{j=0}^{7}\left[\partial_{y_{l j}} E_{l}(y-x), e_{j}, f(y)\right]=0, \quad \forall x \neq y
$$

Indeed, from (3.4) and (3.10), we have

$$
\partial_{y_{l j}} E_{l}(y-x)=\frac{1}{\omega_{8 n}}\left(\frac{\bar{e}_{j}}{|y-x|^{8 n}}-\left(\overline{y_{l}-x_{l}}\right) \frac{8 n\left(y_{l j}-x_{l j}\right)}{|y-x|^{8 n+2}}\right)
$$

so that from the fact that $\left[\bar{e}_{j}, e_{j}, f\right]=0$, we can deduce

$$
\begin{aligned}
& \sum_{l=1}^{n} \sum_{j=0}^{7}\left[\partial_{y_{l j}} E_{l}(y-x), e_{j}, f(y)\right] \\
& \quad=\frac{1}{\omega_{8 n}} \sum_{l=1}^{n} \sum_{j=0}^{7}\left[-\left(\overline{y_{l}-x_{l}}\right) \frac{8 n\left(y_{l j}-x_{l j}\right)}{|y-x|^{8 n+2}}, e_{j}, f(y)\right] .
\end{aligned}
$$

It is evident that the summand can be written as

$$
\left[-\left(\overline{y_{l}-x_{l}}\right) \frac{8 n\left(y_{l j}-x_{l j}\right)}{|y-x|^{8 n+2}}, e_{j}, f(y)\right]=\frac{8 n}{|y-x|^{8 n+2}}\left[\overline{y_{l}-x_{l}},\left(y_{l j}-x_{l j}\right) e_{j}, f(y)\right] .
$$

Since

$$
\sum_{j=0}^{7}\left(y_{l j}-x_{l j}\right) e_{j}=y_{l}-x_{l}
$$


and

$$
\left[\overline{y_{l}-x_{l}}, y_{l}-x_{l}, f(y)\right]=0 \text {, }
$$

we conclude that

$$
\sum_{l=1}^{n} \sum_{j=0}^{7}\left[\partial_{y_{l j}} E_{l}(y-x), e_{j}, f(y)\right]=0
$$

as claimed.

As a result, (3.15) becomes

$$
\mathrm{d}_{y}\left(K_{B M}(y-x) f(y)\right)=\sum_{l=1}^{n} E_{l}(y-x)\left(D_{y_{l}} f(y)\right) \mathrm{d} v_{y} .
$$

Consequently,

$$
\left\{\int_{\partial U}-\int_{\partial B(x, \epsilon)}\right\} K_{B M}(y-x) f(y)=\int_{U \backslash B(x, \epsilon)} \sum_{l=1}^{n} E_{l}(y-x)\left(D_{y_{l}} f(y)\right) \mathrm{d} v_{y} .
$$

By taking the limit process $\epsilon \rightarrow 0$ in the above formula, it is sufficient to show

$$
\begin{aligned}
\lim _{\epsilon \rightarrow 0} \int_{B(x, \epsilon)} \sum_{l=1}^{n} E_{l}(y-x)\left(D_{y_{l}} f(y)\right) \mathrm{d} v_{y} & =0, \\
\lim _{\epsilon \rightarrow 0} \int_{\partial B(x, \epsilon)} K_{B M}(y-x) f(y) & =f(x) .
\end{aligned}
$$

Since $D_{y_{l}} f(y)$ is bounded, it follows that (3.16) holds true.

It remains to show (3.17). For any $y \in \partial B(x, \epsilon)$, we have

$$
|f(y)-f(x)| \leq|x-y| \sup _{\zeta \in \overline{B(x, \epsilon)}}|\nabla f(\zeta)|
$$

so that

$$
\lim _{\epsilon \rightarrow 0} \int_{\partial B(x, \epsilon)} K_{B M}(y-x)(f(y)-f(x))=0 .
$$

Recall that by (3.5)

$$
\mathrm{d} \Sigma_{y_{l}}=\mathrm{d} v_{y_{1}} \wedge \mathrm{d} v_{y_{2}} \wedge \cdots \wedge \mathrm{d} v_{y_{l-1}} \wedge d \sigma_{y_{l}} \wedge \mathrm{d} v_{y_{l+1}} \wedge \cdots \wedge \mathrm{d} v_{y_{n}}
$$

where

$$
\mathrm{d} \sigma_{y_{l}}=\frac{y_{l}-x_{l}}{|y-x|} \mathrm{d} s_{y}
$$


for any $y \in \partial B(x, \epsilon)$.

Since

$$
E_{l}(y-x)=\frac{1}{\omega_{8 n}} \frac{\overline{y_{l}-x_{l}}}{|y-x|^{8 n}}
$$

and $\left[\overline{y_{l}-x_{l}}, y_{l}-x_{l}, f\right]=0$, we have

$$
\left[E_{l}(y-x), \mathrm{d} \Sigma_{y_{l}}, f(x)\right]=0 .
$$

This together with (3.12) yields that

$$
\begin{aligned}
K_{B M}(y-x) f(x) & =\sum_{l=1}^{n} E_{l}(y-x)\left(\mathrm{d} \Sigma_{y_{l}} f(x)\right) \\
& =\left(\sum_{l=1}^{n} E_{l}(y-x) \mathrm{d} \Sigma_{y_{l}}\right) f(x)-\left[E_{l}(y-x), \mathrm{d} \Sigma_{y_{l}}, f(x)\right] \\
& =\left(K_{B M}(y-x)\right) f(x)
\end{aligned}
$$

By Theorem 3.4, we have

$$
\int_{\partial B(x, \epsilon)}\left(K_{B M}(y-x)\right)=1
$$

so that

$$
\int_{\partial B(x, \epsilon)}\left(K_{B M}(y-x)\right) f(x)=f(x)
$$

and (3.17) follows in virtue of (3.18). This finishes the proof.

Remark 3.6 We remark that when $n=1$, Theorem 3.5 recovers the classical CauchyPompeiu formula in octonion analysis [13]

$$
f(x)=\int_{\partial U} E(y-x)\left(d \sigma_{y} f(y)\right)-\int_{U} E(y-x)(D f(y)) \mathrm{d} v_{y} .
$$

Corollary 3.7 Let $U$ be an open bounded set in $\mathbb{R}^{8 n}$ with $C^{2}$ boundary $\partial U$. If $f \in$ $C(\bar{U}, \mathbb{O})$ and $f$ is (octonionic) regular in $U$, then for any $x \in U$,

$$
f(x)=\int_{\partial U} K_{B M}(y-x) f(y) .
$$




\section{Non-homogeneous Cauchy-Riemann Equations}

In this section, we establish the existence theorem for the non-homogeneous CauchyRiemann equations with compact support.

In the setting of quaternions, there are two equivalent characterizations of the compatibility condition for the non-homogeneous Cauchy-Riemann equations. One is expressed in terms of integrals as in (4.2) due to Pertici [14]. The other is given in terms of operators $[5,7,16]$.

Since both of the equivalent compatibility conditions are related to multiplications, it clearly needs modifications due to the non-associativity in the setting of octonions.

For every $1 \leq j \leq n$, let $\Delta_{j}$ be the Laplacian in the $j$-th copy of $\mathbb{O} \cong \mathbb{R}^{8}$ in the Cartesian product $\mathbb{O}^{n}$.

Theorem 4.1 Assume that $n>1$ and $s \geq 3$. Let $g_{1}, g_{2}, \ldots, g_{n} \in C_{0}^{s}\left(\mathbb{O}^{n}\right.$, $\left.\mathbb{O}\right)$. Then the following statements are equivalent:

(1) The system

$$
\left\{\begin{array}{l}
D_{1} f=g_{1} \\
D_{2} f=g_{2} \\
\cdots \\
D_{n} f=g_{n}
\end{array}\right.
$$

admits a solution $f \in C_{0}^{S}\left(\mathbb{O}^{n}, \mathbb{O}\right)$.

(2) For any $p=1, \ldots, n$ and $\left(x_{1}, x^{\prime}\right):=\left(x_{1}, x_{2}, \ldots, x_{n}\right) \in \mathbb{O}^{n}$, there holds

$$
\int_{\mathbb{R}^{8}} \sum_{j=0}^{7} e_{j}\left(E\left(y_{1}\right)\left(\partial_{x_{1 j}} g_{p}-\partial_{x_{p j}} g_{1}\right)\left(y_{1}+x_{1}, x^{\prime}\right)\right) \mathrm{d} v_{y_{1}}=0 .
$$

(3) For any $p, j=1, \ldots, n$, there holds

$$
D_{j}\left(\bar{D}_{p} g_{p}\right)=\Delta_{p} g_{j}
$$

Furthermore, the solution admits an explicit integral expression

$$
f(x)=-\int_{\mathbb{R}^{8}} E\left(y_{1}-x_{1}\right) g_{1}\left(y_{1}, x^{\prime}\right) \mathrm{d} v_{y_{1}}
$$

and vanishes in the unbounded component of $\mathbb{O}^{n} \backslash\left(\operatorname{supp} g_{1} \cup \cdots \cup \operatorname{supp} g_{n}\right)$.

Proof $(1) \Longrightarrow(2)$ : Suppose that system (4.1) admits a solution $f$. We need to show that the compatibility condition (4.2) holds. 
Since $f, \partial_{x_{p j}} f$ all have compact support, we apply Lemma 2.2 with $\partial_{x_{p j}} f$ in place of $f(y)$ to get

$$
\begin{aligned}
0 & =\int_{\mathbb{R}^{8}} e_{j}\left(E\left(y_{1}-x_{1}\right)\left(D_{y_{1}} \partial_{x_{p j}} f\right)\left(y_{1}, x^{\prime}\right)\right) \mathrm{d} v_{y_{1}} \\
& \quad-\int_{\mathbb{R}^{8}} \sum_{i=0}^{7} e_{i}\left(E\left(y_{1}-x_{1}\right)\left(e_{j} \partial_{y_{1 i}} \partial_{x_{p j}} f\right)\left(y_{1}, x^{\prime}\right)\right) \mathrm{d} v_{y_{1}} \\
= & : K_{j}-I_{j} .
\end{aligned}
$$

By assumption, we have $D_{p} f=g_{p}$ so that

$$
K_{j}=\int_{\mathbb{R}^{8}} e_{j}\left(E\left(y_{1}-x_{1}\right)\left(\partial_{x_{p j}} g_{1}\right)\left(y_{1}, x^{\prime}\right)\right) \mathrm{d} v_{y_{1}},
$$

and similarly,

$$
\begin{aligned}
\sum_{j=0}^{7} I_{j} & =\sum_{i=0}^{7} \int_{\mathbb{R}^{8}} e_{i}\left(E\left(y_{1}-x_{1}\right)\left(\partial_{y_{1 i}} D_{p} f\right)\left(y_{1}, x^{\prime}\right)\right) \mathrm{d} v_{y_{1}} \\
& =\sum_{i=0}^{7} \int_{\mathbb{R}^{8}} e_{i}\left(E\left(y_{1}-x_{1}\right)\left(\partial_{y_{1 i}} g_{p}\right)\left(y_{1}, x^{\prime}\right)\right) \mathrm{d} v_{y_{1}} \\
& =\sum_{i=0}^{7} \int_{\mathbb{R}^{8}} e_{i}\left(E\left(y_{1}\right)\left(\partial_{x_{1 i}} g_{p}\right)\left(y_{1}+x_{1}, x^{\prime}\right)\right) \mathrm{d} v_{y_{1}} .
\end{aligned}
$$

Accordingly, from (4.4)-(4.6), we have

$$
\int_{\mathbb{R}^{8}} \sum_{j=0}^{7} e_{j}\left(E\left(y_{1}\right)\left(\partial_{x_{1 j}} g_{p}-\partial_{x_{p j}} g_{1}\right)\left(y_{1}+x_{1}, x^{\prime}\right)\right) \mathrm{d} v_{y_{1}}=0 .
$$

Hence, the compatibility condition holds.

$(2) \Longrightarrow(1)$ : Suppose the integral condition holds. We define

$$
f(x):=-\int_{\mathbb{R}^{8}} E\left(y_{1}-x_{1}\right) g_{1}\left(y_{1}, x^{\prime}\right) \mathrm{d} v_{y_{1}} .
$$

Let $K\left(y_{1}-x_{1}\right)$ be the fundamental solution of $\Delta_{y_{1}}$ in $\mathbb{R}^{8}$, i.e.,

$$
K\left(y_{1}-x_{1}\right)=-\frac{1}{6 \omega_{8}\left|y_{1}-x_{1}\right|^{6}} \text {. }
$$

Since

$$
\bar{D}_{y_{1}} K\left(y_{1}-x_{1}\right)=E\left(y_{1}-x_{1}\right)
$$


we have

$$
\begin{aligned}
f(x) & =-\int_{\mathbb{R}^{8}}\left(\bar{D}_{y_{1}} K\left(y_{1}-x_{1}\right)\right) g_{1}\left(y_{1}, x^{\prime}\right) \mathrm{d} v_{y_{1}} \\
& =\int_{\mathbb{R}^{8}}\left(\bar{D}_{x_{1}} K\left(y_{1}-x_{1}\right)\right) g_{1}\left(y_{1}, x^{\prime}\right) \mathrm{d} v_{y_{1}} .
\end{aligned}
$$

Now we consider the Newtonian potential of $g_{1}$ defined by

$$
w\left(x_{1}, x^{\prime}\right):=\int_{\mathbb{R}^{8}} K\left(y_{1}-x_{1}\right) g_{1}\left(y_{1}, x^{\prime}\right) \mathrm{d} v_{y_{1}} .
$$

By the property of Newtonian potential (see[8, p. 54]), we have

$$
\partial_{x_{1 i}} \int_{\mathbb{R}^{8}} K\left(y_{1}-x_{1}\right) g_{1}\left(y_{1}, x^{\prime}\right) \mathrm{d} v_{y_{1}}=\int_{\mathbb{R}^{8}} \partial_{x_{1 i}} K\left(y_{1}-x_{1}\right) g_{1}\left(y_{1}, x^{\prime}\right) \mathrm{d} v_{y_{1}} .
$$

Since $K\left(y_{1}-x_{1}\right)$ are scalar-valued function, we obtain that

$$
f(x)=\bar{D}_{x_{1}} \int_{\mathbb{R}^{8}} K\left(y_{1}-x_{1}\right) g_{1}\left(y_{1}, x^{\prime}\right) \mathrm{d} v_{y_{1}} .
$$

That is

$$
f(x)=\bar{D}_{1} w\left(x_{1}, x^{\prime}\right) .
$$

Hence,

$$
D_{1} f(x)=D_{1}\left(\bar{D}_{1} w\left(x_{1}, x^{\prime}\right)\right) .
$$

By the associator of three octonions

$$
\left.D_{1}\left(\bar{D}_{1} w\left(x_{1}, x^{\prime}\right)\right)=\left(D_{1} \bar{D}_{1}\right) w\left(x_{1}, x^{\prime}\right)\right)-\left[D_{1}, \bar{D}_{1}, w\left(x_{1}, x^{\prime}\right)\right]
$$

and

$$
\left[D_{1}, \bar{D}_{1}, w\left(x_{1}, x^{\prime}\right)\right]=0
$$

we get that

$$
D_{1} f(x)=\left(D_{1} \bar{D}_{1}\right) w\left(x_{1}, x^{\prime}\right)=\Delta_{1} w\left(x_{1}, x^{\prime}\right) .
$$

Since the Newtonian potential $w\left(x_{1}, x^{\prime}\right)$ of $g_{1}\left(x_{1}, x^{\prime}\right)$ is a solution of the Poisson equation, i.e.,

$$
\Delta_{1} w\left(x_{1}, x^{\prime}\right)=g_{1}\left(x_{1}, x^{\prime}\right)
$$


we have

$$
D_{1} f(x)=g_{1}(x)
$$

Now for any $p=2, \ldots, n$, by differentiating under the sign of integration, we obtain

$$
\begin{aligned}
D_{x_{p}} f(x) & =-\int_{\mathbb{R}^{8}} D_{x_{p}}\left(E\left(y_{1}-x_{1}\right) g_{1}\left(y_{1}, x^{\prime}\right)\right) \mathrm{d} v_{y_{1}} \\
& =-\int_{\mathbb{R}^{8}} \sum_{j=0}^{7} e_{j}\left(E\left(y_{1}-x_{1}\right)\left(\partial_{x_{p j}} g_{1}\left(y_{1}, x^{\prime}\right)\right)\right) \mathrm{d} v_{y_{1}} .
\end{aligned}
$$

By assumption (4.2), the right-hand side of the above integral is equal to

$$
\begin{aligned}
& -\int_{\mathbb{R}^{8}} \sum_{j=0}^{7} e_{j}\left(E\left(y_{1}-x_{1}\right)\left(\partial_{x_{1 j}} g_{p}\left(y_{1}, x^{\prime}\right)\right)\right) \mathrm{d} v_{y_{1}} \\
& =-\int_{\mathbb{R}^{8}} \sum_{j=0}^{7} e_{j}\left(E\left(y_{1}-x_{1}\right)\left(\partial_{y_{1 j}} g_{p}\left(y_{1}, x^{\prime}\right)\right)\right) \mathrm{d} v_{y_{1}} .
\end{aligned}
$$

Since $g_{p} \in C^{s}\left(\mathbb{O}^{n}, \mathbb{O}\right)$ has compact support and $s \geq 2$, it follows from Lemma 2.1 that

$$
-\int_{\mathbb{R}^{8}} \sum_{j=0}^{7} e_{j}\left(E\left(y_{1}-x_{1}\right)\left(\partial_{y_{1 j}} g_{p}\left(y_{1}, x^{\prime}\right)\right)\right) \mathrm{d} v_{y_{1}}=g_{p}\left(x_{1}, x^{\prime}\right) .
$$

Consequently, we have

$$
D_{p} f=g_{p}, \quad p=1,2, \cdots n \text {. }
$$

$(1) \Longrightarrow(3)$ : If that system (4.1) admits a solution $f$, i.e., $D_{p} f=g_{p}$, we know

$$
D_{j}\left(\bar{D}_{p} g_{p}\right)=D_{j}\left(\bar{D}_{p}\left(D_{p} f\right)\right)=D_{j}\left(\Delta_{p} f\right)=\Delta_{p} D_{j} f=\Delta_{p} g_{j}
$$

since $\bar{D}_{p}\left(D_{p} f\right)=\left(\bar{D}_{p} D_{p}\right) f=\Delta_{p} f$ and $\Delta_{p}$ are scalar-valued operator.

(3) $\Longrightarrow(1)$ : We need to verify that the function $f$ defined in (4.7) satisfies equations (4.1). With the same approach as in (4.10), we can show that $D_{1} f=g_{1}$. It remains to show that $D_{j} f=g_{j}$ for any $j=2, \ldots, n$. The same reason as in (4.8) shows that

$$
f(x)=\bar{D}_{x_{1}} \int_{\mathbb{R}^{8}} K\left(y_{1}-x_{1}\right) g_{1}\left(y_{1}, x^{\prime}\right) \mathrm{d} v_{y_{1}} .
$$


Since $\Delta_{p}$ for $p \in\{2, \ldots, n\}$ and $K\left(y_{1}-x_{1}\right)$ are scalar-valued, and $g_{1}$ has compact support, we apply $\Delta_{p}$ to both sides in the above formula to get

$$
\Delta_{p} f(x)=\bar{D}_{x_{1}} \int_{\mathbb{R}^{8}} K\left(y_{1}-x_{1}\right)\left(\Delta_{p} g_{1}\left(y_{1}, x^{\prime}\right)\right) \mathrm{d} v_{y_{1}}
$$

Since $D_{1}\left(\bar{D}_{p} g_{p}\right)=\Delta_{p} g_{1}$ by assumption, we thus get

$$
\Delta_{p} f(x)=\bar{D}_{x_{1}} \int_{\mathbb{R}^{8}} K\left(y_{1}-x_{1}\right)\left\{\left(D_{1}\left(\bar{D}_{p} g_{p}\right)\right)\left(y_{1}, x^{\prime}\right)\right\} \mathrm{d} v_{y_{1}} .
$$

Notice that $K\left(y_{1}-x_{1}\right)$ is scalar-valued, we have

$$
K\left(y_{1}-x_{1}\right) D_{y_{1}}=-K\left(y_{1}-x_{1}\right) D_{x_{1}}=-D_{x_{1}} K\left(y_{1}-x_{1}\right)
$$

so that

$$
\begin{aligned}
\Delta_{p} f(x) & =-\bar{D}_{x_{1}} \int_{\mathbb{R}^{8}}\left(K\left(y_{1}-x_{1}\right) D_{y_{1}}\right)\left(\bar{D}_{p} g_{p}\left(y_{1}, x^{\prime}\right)\right) \mathrm{d} v_{y_{1}} \\
& =\bar{D}_{x_{1}} \int_{\mathbb{R}^{8}}\left(D_{x_{1}} K\left(y_{1}-x_{1}\right)\right)\left(\bar{D}_{p} g_{p}\left(y_{1}, x^{\prime}\right)\right) \mathrm{d} v_{y_{1}} \\
& =\bar{D}_{x_{1}}\left\{D_{x_{1}} \int_{\mathbb{R}^{8}} K\left(y_{1}-x_{1}\right)\left(\bar{D}_{p} g_{p}\left(y_{1}, x^{\prime}\right)\right) \mathrm{d} v_{y_{1}}\right\} .
\end{aligned}
$$

Due to $\left[\bar{D}_{1}, D_{1}, \cdot\right]=0$, we have

$$
\begin{aligned}
\Delta_{p} f(x) & =\left(\bar{D}_{x_{1}} D_{x_{1}}\right) \int_{\mathbb{R}^{8}} K\left(y_{1}-x_{1}\right)\left(\bar{D}_{p} g_{p}\left(y_{1}, x^{\prime}\right)\right) \mathrm{d} v_{y_{1}} \\
& =\bar{D}_{p} g_{p}(x)
\end{aligned}
$$

The last step uses the facts that $\bar{D}_{x_{1}} D_{x_{1}}=\Delta_{x_{1}}$ and that the last integral is the Newtonian potential of $\bar{D}_{p} g_{p}$. Thus, $\Delta_{p} f=\bar{D}_{p} g_{p}$ for any $p=1, \ldots, n$. This together with the assumption that $D_{j}\left(\bar{D}_{p} g_{p}\right)=\Delta_{p} g_{j}$ shows

$$
\Delta_{p}\left(D_{j} f-g_{j}\right)=D_{j} \Delta_{p} f-\Delta_{p} g_{j}=D_{j}\left(\bar{D}_{p} g_{p}\right)-\Delta_{p} g_{j}=0
$$

Summing up in $p$ shows that $D_{j} f-g_{j}$ is harmonic in $\mathbb{O}^{n}$ for each $j$. By construction in (4.7), $f$ has compact support when $x^{\prime}$ is large. Since the harmonic function $D_{j} f-g_{j}$ vanishes in an open set, we thus conclude that $D_{j} f=g_{j}$ in $\mathbb{O}^{n}$ for each $j$.

Finally, we come to evaluate the support of the solution $f$ to systems (4.1). By virtue of (4.1), we have

$$
\left.D_{p} f\right|_{\mathbb{O}^{n} \backslash\left(\operatorname{supp}_{1} \cup \ldots \cup \operatorname{supp} g_{n}\right)}=0 .
$$


Therefore, $f$ is regular and thus harmonic outside $\operatorname{supp} g_{1} \cup \cdots \cup \operatorname{supp} g_{n}$. From (4.7), we find $f\left(x_{1}, x_{2}, \ldots, x_{n}\right)=0$ provided $\left|x_{2}\right|^{2}+\cdots+\left|x_{n}\right|^{2}$ large enough. The identity principle for harmonic functions thus implies that $f$ vanishes in the unbounded component of $\mathbb{O}^{n} \backslash\left(\operatorname{supp} g_{1} \cup \cdots \cup \operatorname{supp} g_{n}\right)$. This completes the proof.

Remark 4.2 We remark that the existence theorem of the non-homogeneous CauchyRiemann equations plays an eminent role in the theory of several complex variables $[10,11]$. The remarkable difference between the theory in several octonionic variables and that of several complex variables lies at its non-commutativity and nonassociativity

$$
D_{i} D_{j} \neq D_{j} D_{i}, \quad\left(D_{i} D_{j}\right) f \neq D_{i}\left(D_{j} f\right)
$$

This means that unlike in the case of several complex variables, the conditions

$$
D_{i} g_{j}=D_{j} g_{i}
$$

fail to become the compatibility conditions of the non-homogeneous Cauchy-Riemann equations (4.1).

\section{Hartogs Theorem}

In virtue of the existence of compactly-supported solution for the system of nonhomogeneous Cauchy-Riemann equations, we can deduce Hartogs theorem of several octonionic variables.

Theorem 5.1 (Hartogs) Let $U$ be a connected open set in $\mathbb{O}^{n} \cong \mathbb{R}^{8 n}$ with $n>1$. Let $M \subset U$ be a compact set such that $U \backslash M$ is connected. Then every regular function as defined in Definition 3.1 in $U \backslash M$ can be extended to a regular function on $U$.

Proof Let $f$ be a regular function in $U \backslash M$. Take a function $\varphi \in C^{\infty}(U, \mathbb{R})$ with compact support such that $\varphi=1$ in a neighborhood of $M$ and set

$$
f_{0}:=(1-\varphi) f \quad \text { in } U \text {. }
$$

Obviously, $f_{0} \in C^{\infty}(U, \mathbb{O})$ and regular in $U \backslash \operatorname{supp} \varphi$. Let us set

$$
h_{p}:= \begin{cases}D_{p} f_{0}, & \text { in } U, \\ 0, & \text { in } \mathbb{O}^{n} \backslash U .\end{cases}
$$

It can be rewritten as

$$
h_{p}= \begin{cases}D_{p} f_{0}, & \text { in } \operatorname{supp} \varphi, \\ 0, & \text { in } \mathbb{O}^{n} \backslash \operatorname{supp} \varphi\end{cases}
$$

Clearly, $h_{p} \in C_{0}^{\infty}\left(\mathbb{O}^{n}, \mathbb{O}\right)$ and $\operatorname{supp} h_{p} \subset \operatorname{supp} \varphi$. 
By (5.2), we know that in $U$

$$
D_{j}\left(\bar{D}_{p} h_{p}\right)=D_{j}\left(\bar{D}_{p}\left(D_{p} f_{0}\right)\right)=D_{j}\left(\Delta_{p} f_{0}\right)=\Delta_{p}\left(D_{j} f_{0}\right)=\Delta_{p} h_{j} .
$$

It is clear that there holds $D_{j}\left(\bar{D}_{p} h_{p}\right)=\Delta_{p} h_{j}$ outside $U$. This implies that functions $h_{p}$ satisfy the compatibility conditions in (4.3). By Theorem 4.1, the system

$$
D_{p} g=h_{p}, \quad p=1,2, \ldots, n
$$

has a solution $g \in C_{0}^{\infty}\left(\mathbb{O}^{n}, \mathbb{O}\right)$, and $g$ vanishes on the unbounded component of $\mathbb{O}^{n} \backslash\left(\operatorname{supp} h_{1} \cup \cdots \cup \operatorname{supp} h_{n}\right)$. From (5.3), we have

$$
\operatorname{supp} h_{p} \subset \operatorname{supp} \varphi, \quad \forall p=1, \ldots, n
$$

so that

$$
\mathbb{O}^{n} \backslash \operatorname{supp} \varphi \subset \mathbb{O}^{n} \backslash\left(\operatorname{supp} h_{1} \cup \cdots \cup \operatorname{supp} h_{n}\right) .
$$

Consequently, $g$ vanishes on the unbounded component $M_{0}$ of $\mathbb{O}^{n} \backslash \operatorname{supp} \varphi$.

We now set

$$
\tilde{f}:=f_{0}-g .
$$

From (5.2), we have $D_{p} f_{0}=h_{p}$ in $U$ so that $\tilde{f}$ is regular in $U$.

By construction, we see

$$
\left.g\right|_{M_{0}}=0,\left.\quad\left(f-f_{0}\right)\right|_{U \backslash \operatorname{supp} \varphi}=0, \quad M_{0} \subset \mathbb{O}^{n} \backslash \operatorname{supp} \varphi .
$$

This implies that $\tilde{f}$ coincides with $f$ on $M_{0} \cap U$.

Since

$$
M_{0} \cap U \subset U \backslash \operatorname{supp} \varphi \subset U \backslash M
$$

and $U \backslash M$ is connected, it follows from the identity principle that $\tilde{f}$ coincides with $f$ on $U \backslash M$ so that $\tilde{f}$ is the regular extension of $f$.

Acknowledgments This work was supported by the NNSF of China (11071230), RFDP (20123402110068).

\section{References}

1. Adams, W.W., Berenstein, C.A., Loustaunau, P., Sabadini, I., Struppa, D.C.: Regular functions of several quaternionic variables and the Cauchy-Fueter complex. J. Geom. Anal. 9, 1-15 (1999)

2. Adams, W.W., Loustaunau, P.: Analysis of the module determining the properties of regular funcions of several quaternionic variables. Pacific J. 196, 1-15 (2001)

3. Baez, J.C.: The octonions. Bull. Amer. Math. Soc. 39, 145-205 (2002) 
4. Bossard, G.: Octonionic black holes. arXiv:1203.0530

5. Bureš, J., Damiano, A., Sabadini, I.: Explicit resolutions for several Fueter operators. J. Geom. Phys. 57, 765-775 (2007)

6. Colombo, F., Sabadini, I., Sommen, F., Struppa, D C.: Analysis of Dirac Systems and Computational Algebra. Progress in Mathematical Physics, vol. 39. Birkhäuser, Boston (2004)

7. Colombo, F., Souček, V., Struppa, D.C.: Invariant resolutions for several Fueter operators. J. Geom. Phys. 56, 1175-1191 (2006)

8. Gilbarg, D., Trudinger, N.S.: Elliptic Partial Differential Equations of Second Order. Springer-Verlag, Berlin (2001)

9. Harvey, F.Reese, Lawson Jr, HBlaine: On boundaries of complex analytic varieties. I. Ann. Math. 102(2), 223-290 (1975)

10. Hörmander, L.: An Introduction to Complex Analysis in Several Variables. North-Holland Mathematical Library, vol. 7. North-Holland Publishing Co, Amsterdam (1990)

11. Krantz, S.G.: Function Theory of Several Complex Variables. AMS Chelsea Publishing, Providence (2001)

12. Kytmanov, A.: The Bochner-Martinelli Integral and its Applications. Birkhäuser Verlag, Basel (1995)

13. Li, X.M., Peng, L.Z.: The Cauchy integral formulas on the octonions. Bull. Belg. Math. Soc. Simon Stevin 9, 47-64 (2002)

14. Pertici, D.: Regular functions of several quaternionic variables. Ann. Mat. Pura Appl. 151(4), 39-65 (1988)

15. Ren, G. B., Wang, H. Y.: Theory of several Clifford variables: Bochner-Martinelli formula and Hartogs theorem, submitted

16. Wang, W.: On non-homogeneous Cauchy-Fueter equations and Hartogs phenomenon in several quaternionic variables. J. Geom. Phys. 58, 1203-1210 (2008) 